\title{
Degree of freedom analysis of hexapod wall-climbing robot
}

\author{
Chuntai Ye ${ }^{\mathrm{a}}$, Yinlong Yuan ${ }^{\mathrm{b}}$ and Wu Wei \\ School of Automation Science \& Engineering, South China University of Technology, Guangzhou \\ Guangdong 510641, China \\ ayect1992@163.com, 'buanyinlong2009@163.com, *Corresponding Author Email: \\ eeweiwu@126.com
}

Keywords: hexapod robot, spinor theory, degree of freedom analysis, symmetrically initial states.

\begin{abstract}
The knowledge of degree of freedom (referred as DOF) is indispensable before a parallel mechanism is designed, but the traditional Grubler-Kutzbach formula (referred as G-K formula) which is widely used is not appropriate for complicated mechanism, especially the hexapod robots. In order to derive the kinematics model of the robot, the motion of single branch and the corresponding spinors is derived based on spinor theory. The constraint spinors of each branch are integrated and the allowed movement matrix is determined. And then, the DOF of the robot in a certain initial state is derived from the rank of the matrix. Furthermore, the DOF analysis under symmetrically initial states is discussed in detail.
\end{abstract}

\section{Introduction}

As a basic problem of mechanism analysis, DOF must be first determined according to specific applications. The traditional G-K formula has been used to calculate the DOF for a long time, but is no longer applicable because of the development of mechanisms ${ }^{[1-3]}$, especially for analysis of parallel mechanisms and spatial mechanisms ${ }^{[4]}$. The latter of this paper shows that a complete position analysis is indispensable to completely analyze the DOF of parallel mechanisms.

In the early twenty-first century, many significant calculation method like analysis methods based on group theory and Lie algebras ${ }^{[5-6]}$ were presented. Furthermore, calculation methods based on spinor theory, which developed by Zhao Jingshan ${ }^{[7]}$, Huang Zhen ${ }^{[8]}$, Gosselin ${ }^{[9]}$ as representatives, is very effective in analyzing spatial robot and parallel mechanisms ${ }^{[10-13]}$ and have been increasingly recognized. By representing the basic constraint relations under the reciprocal principle of spinor theory, this method present an analysis method of DOF for modern parallel mechanisms, which proves its great universality and practicality.

As one kind of special parallel robots, hexapod wall-climbing robots have six movable legs. The DOF of the robots changes under different initial states. Usually branch structures of mechanisms are designed to be symmetrical to ensure the universality of application. And the analysis of DOF under symmetrically initial states has significant sense, because DOF information under different initial states have guiding effect on the trajectory planning. DOF analysis under different symmetrically initial states of hexapod robot are discussed in detail based on spinor theory in this paper.

\section{Motion of Rigid Body and Lie Groups\& Screw Theory}

According to Chasles's theorem, any motion of a rigid body can be presented by spinor motion, that is, by rotating around an axis and then translating along an axis. As shown in Figure 1, any configuration of a rigid body relative to fixed coordinate could be determined by position ( $\boldsymbol{P} \in \boldsymbol{R}^{3}$ ) and orientation ( $\boldsymbol{R} \in S O(3)$ ).

Therefore configuration of a rigid body can be expressed as the product space $S E(3)$.

$S E(3)=\left\{(\boldsymbol{R}, \boldsymbol{P}): \boldsymbol{R} \in S O(3), \boldsymbol{P} \in \boldsymbol{R}^{3}\right\}=S O(3) \otimes \boldsymbol{R}^{3}$. Where $S O(3)$ is a three dimensional rotation group. 


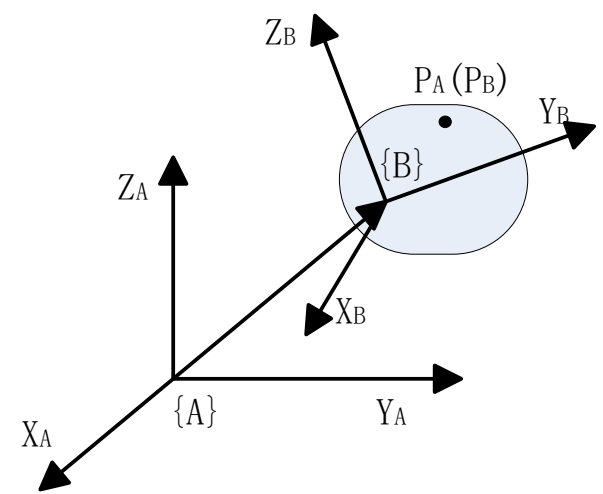

Define an expression:

Figure 1:Rigid body mechanism presented by spinors

$$
\operatorname{se}(3)=\left\{\hat{\xi} \mid \hat{\xi} \mapsto(\hat{\omega} ; v): \hat{\omega} \in \operatorname{so}(3), v \in \boldsymbol{R}^{3}\right\}
$$

Where $\hat{\xi} \in s e(3)$ is Lie algebraic expression of European group $S E(3)$, which mean the generalized instantaneous velocity of rigid body. $\hat{\omega} \in s o(3)$ is the Lie algebraic expressions of the European group $S O(3)$ and $s o(3)=\left\{\hat{\omega} \in \boldsymbol{R}^{3}: \hat{\omega}^{T}=-\hat{\omega}\right\}$, where $\hat{\xi}$ is the corresponding motion spinor of joint(twist).

There is an exponential mapping relationship between the elements of se(3) and the elements of $S E(3)$ :

$$
e^{\theta \hat{\xi}}=\left(\begin{array}{cc}
e^{\theta \hat{\omega}} & \left(\boldsymbol{I}-e^{\theta \hat{\omega}}\right)(\omega-v)+\theta \omega \omega^{T} v \\
0 & 1
\end{array}\right)=\left(\begin{array}{cc}
\boldsymbol{R} & \boldsymbol{P} \\
0 & 1
\end{array}\right)
$$

Based on spinor theory, the process of establishing variety coordinate systems is simplified and the whole system consists of two coordinate systems: the inertial coordinate system $\{\boldsymbol{S}\}$ and the tool coordinate system $\{\boldsymbol{T}\}$. Thus the analysis process based on spinor theory is simpler than traditional methods'. The product of exponential formula (POE) is one of the most representative formula of the kinematic formulas.

$$
g_{S T}(\theta)=e^{\theta_{1} \hat{\xi}_{1}} e^{\theta_{2} \hat{\xi}_{2}} \cdots e^{\theta_{i} \hat{\xi}_{i}} \ldots e^{\theta_{n} \hat{\xi}_{n}} g_{S T}(0)
$$

Where $\xi_{i}$ is spinor coordinate of unit movement of $i$ th joint under initial configuration $g_{S T}(0)$ relative to inertial coordinate system. The following of the paper is to analyze the hexapod wall-climbing robot based on the Lie groups and spinor theory.

\section{Kinematics Model}

Figure 2 shows mechanism model of the laboratory wall-climbing robot named ML6-QJ-I. The joints of hip, knee, ankle and foot are revolute joints. The foot tip is connected with a sucker by a spherical joint. Each leg has the same structure and size. The initial state when six suckers are on the ground follows the principle of symmetry. The basic reference frame (o-xyz) is built at the geometric center of the six suckers $B_{1} B_{2} B_{3} B_{4} B_{5} B_{6}$ connected to tips of legs. In the same way, the local reference frame of end effector $\left(o_{c}-x_{c} y_{c} z_{c}\right)$ is built at the geometric center of the body platform $A_{1} A_{2} A_{3} A_{4} A_{5} A_{6}$.

When $M N$ is the axisymmetric line of initial states, define:

$$
\begin{aligned}
& \alpha=\angle M_{1} A_{1} A_{6}=\angle M_{6} A_{6} A_{1} \\
& \beta=\angle M_{2} A_{2} A_{1}=\angle M_{5} A_{5} A_{6} \\
& \gamma=\angle M_{3} A_{3} A_{2}=\angle M_{4} A_{4} A_{5}
\end{aligned}
$$

When line $O O_{c}$ is symmetrical axis of initial states, define:

$$
\vartheta=\angle M_{1} A_{1} A_{2}=\angle M_{2} A_{2} A_{3}=\angle M_{3} A_{3} A_{4}=\angle M_{4} A_{4} A_{5}=\angle M_{5} A_{5} A_{6}=\angle M_{6} A_{6} A_{1}
$$

Where $\partial, \beta, \gamma, \vartheta \in\left(60^{0}, 180^{\circ}\right)$. 


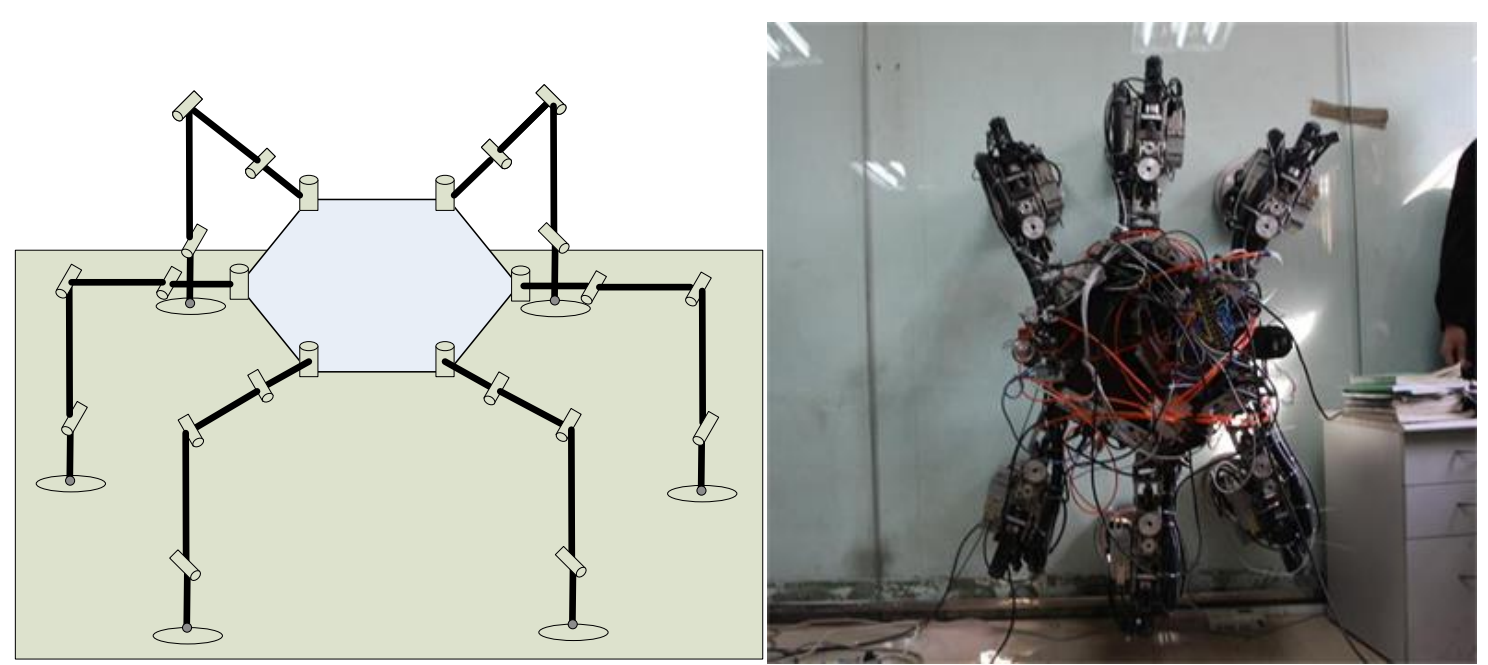

Figure 2:Mechanism model of hexapod wall-climbing robot ML6-QJ-I

Generalized instantaneous speed of foot tip is determined by the configuration of foot tip: $g_{S T}(\theta) \in S E(3)$.

$$
\hat{V}_{S T}^{s}=g_{S T}^{-1}(\theta) \dot{g}_{S T}(\theta)=\sum_{i=1}^{n}\left(g_{S T}^{-1}(\theta) \frac{\partial g_{S T}}{\partial \theta_{i}}\right) \dot{\theta}_{i}
$$

The corresponding spinor coordinates can be expressed as:

$$
V_{S T}^{S}=\sum_{i=1}^{n}\left(g_{S T}^{-1}(\theta) \frac{\partial g_{S T}}{\partial \theta_{i}}\right)^{\vee} \dot{\theta}_{i}=\boldsymbol{J}_{S T}^{S}(\theta) \dot{\theta}_{\boldsymbol{i}}
$$

And $\xi_{i}^{\prime}=A d_{\left(e^{-\theta_{n} \hat{n}_{e}} e^{-\theta_{n-1} \hat{\xi}_{n-1}-\ldots} e^{-\theta_{i+1} \hat{\xi}_{i+1}}\right)} \xi_{i}$,

$\boldsymbol{J}_{S T}^{S}(\theta)=\left(\xi_{1}^{\prime} \xi_{2}^{\prime} \cdots \xi_{\boldsymbol{n}}^{\prime}\right), \quad \dot{\theta}_{\boldsymbol{i}}=\left(\dot{\theta}_{1} \dot{\theta}_{2} \cdots \dot{\theta}_{\boldsymbol{n}}\right)^{T}$. Where $\boldsymbol{J}_{S T}^{S}(\theta)$ is Jacobian matrix of spatial velocity; $\dot{\theta}_{\boldsymbol{i}}$ is the velocity of join ' $\mathrm{i}$ '; $\xi_{i}^{\prime}$ is the motion spinor of coordinate system of joint ' $\mathrm{i}$ ' to current configuration, which is the basis of kinematics of the robot. The DOF is determined by states of spinor motion of foot tips allowed in the process.

$$
M=\operatorname{rank}\left(\xi_{\mathrm{A}_{1} \mathrm{~A}_{2} \mathrm{~A}_{3} \mathrm{~A}_{4} \mathrm{~A}_{3} \mathrm{~A}_{6}}\right)
$$

\section{Analysis of DOF}

The coordinate system established is shown in figure 3 and the spherical joint of foot tip can be decomposed into two mutually perpendicular revolute joints in practical situations. The rotation angle of them are $\theta_{1} 、 \theta_{2}$ respectively. The joints of foot, ankle, knee and hip are defined as $\theta_{3} 、 \theta_{4} 、 \theta_{5}$ 、 $\theta_{6}$ respectively. Obviously, rotation range changes under different initial states of the robot.

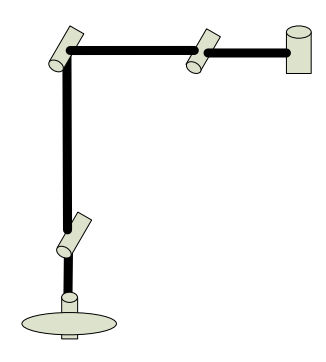

Figure 3:Transformation model of single-branched structure

\section{Analysis of DOF under Initial States of Axis-symmetry}

When $M N$ is the axisymmetric line of initial states, the length of the body platform is defined as $R_{1}$, and he projected length of $B_{i} A_{i}$ on plane oxy is $R_{2}$. The coordinate values of foot tips are 


$$
\begin{array}{lr}
B_{1}\left(-\frac{\sqrt{3}}{2} R_{1}-R_{2} \sin \alpha,-\frac{1}{2} R_{1}+R_{2} \cos \alpha, 0\right) & B_{2}\left(-R_{2} \sin \left(\beta-120^{0}\right),-R_{1}-R_{2} \cos (\beta-120), 0\right) \\
B_{3}\left(\frac{\sqrt{3}}{2} R_{1}+R_{2} \sin \left(\gamma-60^{\circ}\right),-\frac{1}{2} R_{1}-R_{2} \cos (\gamma-60), 0\right) & B_{4}\left(\frac{\sqrt{3}}{2} R_{1}+R_{2} \sin \left(\gamma-60^{\circ}\right), \frac{1}{2} R_{1}+R_{2} \cos (\gamma-60), 0\right) \\
B_{5}\left(-R_{2} \sin \left(\beta-120^{\circ}\right), R_{1}+R_{2} \cos (\beta-120), 0\right) & B_{6}\left(-\frac{\sqrt{3}}{2} R_{1}-R_{2} \sin \alpha, \frac{1}{2} R_{1}-R_{2} \cos \alpha, 0\right) .
\end{array}
$$

The motion spinor of leg ' 1 ' is first analyzed. The matrix of motion spinor of leg ' 1 ' is given below based on the analysis above:

$$
\xi_{B_{1} A_{1}}^{\omega}=\left[\begin{array}{llllll}
\xi_{1}^{1} & \xi_{2}^{1} & \xi_{3}^{1} & \xi_{4}^{1} & \xi_{5}^{1} & \xi_{6}^{1}
\end{array}\right]
$$

where

$$
\left\{\begin{array}{l}
\xi_{1}^{1}=\left[\begin{array}{llllll}
0 & 0 & 1 & s_{01}^{1} M_{1} & -c_{01}^{1} M_{1} & 0
\end{array}\right]^{T} \\
\xi_{2}^{1}=\left[\begin{array}{llllll}
s_{01}^{1} & -c_{01}^{1} & 0 & -c_{01}^{1} N_{1} & -s_{01}^{1} N_{1} & -M_{1}
\end{array}\right]^{T} \\
\xi_{3}^{1}=\left[\begin{array}{llllll}
s_{01}^{1} & -c_{01}^{1} & 0 & -c_{01}^{1}\left(N_{1}-m_{1} c_{2}\right) & -s_{01}^{1}\left(N_{1}-m_{1} c_{2}\right) & -\left(M_{1}-m_{1} s_{2}^{1}\right)
\end{array}\right]^{T} \\
\xi_{4}^{1}=\left[\begin{array}{llllll}
s_{01}^{1} & -c_{01}^{1} & 0 & -c_{01}^{1}\left(N_{1}-m_{1} c_{2}^{1}-m_{2} c_{23}^{1}\right) & -s_{01}^{1}\left(N_{1}-m_{1} c_{2}^{1}-m_{2} c_{23}^{1}\right) & -\left(M_{1}-m_{1} s_{2}^{1}-m_{2} s_{23}^{1}\right)
\end{array}\right]^{T} \\
\xi_{5}^{1}=\left[\begin{array}{llllll}
s_{01}^{1} & -c_{01}^{1} & 0 & -c_{01}^{1} m_{4} s_{2345}^{1} & -s_{01}^{1} m_{4} s_{2345}^{1} & m_{4} c_{2345}^{1}
\end{array}\right]^{T} \\
\xi_{6}^{1}=\left[\begin{array}{llllll}
-c_{01}^{1} s_{2345}^{1} & -s_{01}^{1} s_{2345}^{1} & c_{2345}^{1} & 0 & 0 & 0
\end{array}\right]^{T}
\end{array}\right.
$$
below.

According to reciprocal principle of spinor theory, the constraint spinor of foot tip ' 1 ' is given

$$
\xi_{B_{1} A_{1}}^{\tau}=k_{1}\left[\begin{array}{llllll}
-s_{01}^{1} s_{2345}^{1} & c_{01}^{1} s_{2345}^{1} & 0 & c_{01}^{1} c_{2345}^{1} M_{1} & s_{01}^{1} c_{2345}^{1} M_{1} & s_{2345}^{1} M_{1}
\end{array}\right]^{T}
$$

Where $k_{1}$ is an arbitrary non-zero constant.

In the same way, constraint spinors of other five foot tips relative to reference frame are determined as follows.

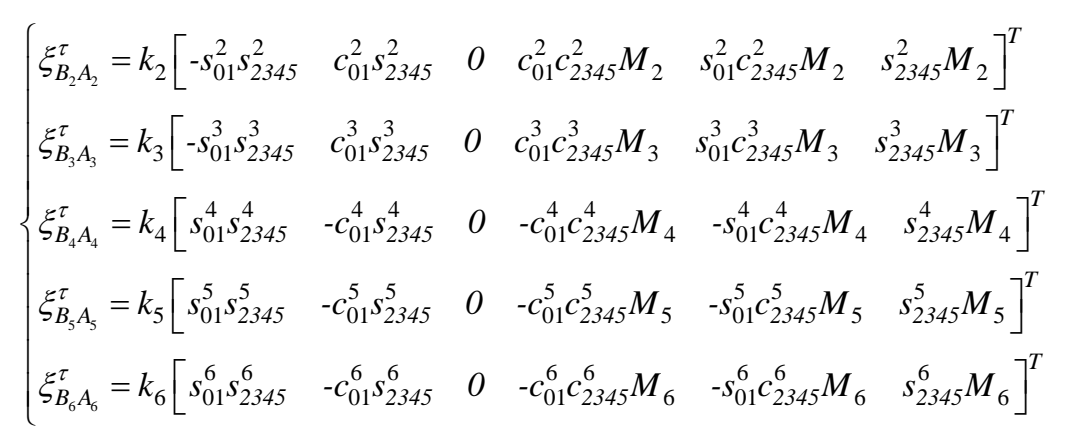

Where $\quad c_{i}^{n}=\cos \theta_{i}^{n} \quad, \quad s_{i}^{n}=\sin \theta_{i}^{n} \quad, \quad c_{i j}^{n}=\cos \left(\theta_{i}^{n}+\theta_{i}^{n}\right) \quad, \quad c_{i j k}^{n}=\cos \left(\theta_{i}^{n}+\theta_{j}^{n}+\theta_{k}^{n}\right) \quad$, $s_{i j k}^{n}=\sin \left(\theta_{i}^{n}+\theta_{j}^{n}+\theta_{k}^{n}\right), s_{i j}^{n}=\sin \left(\theta_{i}^{n}+\theta_{j}^{n}\right), N_{n}=m_{1} s_{2}^{n}+m_{2} s_{23}^{n}-m_{3} c_{234}^{n}-m_{4} c_{2345}^{n}, M_{n}=m_{1} c_{2}^{n}+m_{2} c_{23}^{n}+m_{3} s_{234}^{n}+m_{4} s_{2345}^{n}$, $\theta_{01}^{1}=\alpha-90^{0}+\theta_{1}^{1}, \theta_{01}^{2}=\beta-30^{0}+\theta_{1}^{2}, \theta_{01}^{3}=\gamma+30^{0}+\theta_{1}^{3}, \theta_{01}^{4}=150^{0}-\gamma+\theta_{1}^{4}, \theta_{01}^{5}=210^{0}-\beta+\theta_{1}^{5}, \theta_{01}^{6}=270^{0}-\alpha+\theta_{1}^{6}$ 。

Therefore the constraint of body platform $A_{1} A_{2} A_{3} A_{4} A_{5} A_{6}$ of the robot is:

$$
\xi_{A_{1} A_{2} A_{3} A_{4} A_{5} A_{6}}^{r}=\left[\begin{array}{llllll}
\xi_{B_{1} A_{1}}^{\tau} & \xi_{B_{2} A_{2}}^{\tau} & \xi_{B_{3} A_{3}}^{\tau} & \xi_{B_{4} A_{4}}^{\tau} & \xi_{B_{5}}^{\tau} A_{5} & \xi_{B_{6}}^{\tau} A_{6}
\end{array}\right]
$$

Clearly, $\xi_{A_{1} A_{2} A_{3} A_{4} A_{3} A_{6}}$ is related to the initial state and rotational angle of each joint. The corresponding analysis of DOF is shown as follows.

Since the directions of spinors of end joints connected with platform $A_{1} A_{2} A_{3} A_{4} A_{5} A_{6}$ are the same in movement and satisfy the constraint: $\omega_{6}^{1}=\omega_{6}^{2}=\omega_{6}^{3}=\omega_{6}^{4}=\omega_{6}^{5}=\omega_{6}^{6} \quad$. That is: 


$$
\left\{\begin{array}{l}
-c_{01}^{1} s_{2345}^{1}=-c_{01}^{2} s_{2345}^{2}=-c_{01}^{3} s_{2345}^{3}=c_{01}^{4} s_{2345}^{4}=c_{01}^{5} s_{2345}^{5}=c_{01}^{6} s_{2345}^{6} \\
-s_{01}^{1} 1_{2345}^{1}=-s_{01}^{2} s_{2345}^{2}=-s_{01}^{3} s_{2345}^{3}=s_{01}^{4} s_{2345}^{4}=s_{01}^{5} 5_{2345}^{5}=s_{01}^{6} s_{2345}^{6} \\
c_{2345}^{1}=c_{2345}^{2}=c_{2345}^{3}=c_{2345}^{4}=c_{2345}^{5}=c_{2345}^{6}
\end{array}\right.
$$

Conclusions can be obtained as follows:

(1) The body platform has three DOF when $s_{2345}^{i} \neq 0$.

Demonstration: If $s_{2345}^{i} \neq 0$, then $c_{2345}^{i}=c_{2345}^{j}(i \neq j)$ and $s_{2345}^{i}= \pm s_{2345}^{j}(i \neq j)$. Equations are satisfied as shown below:

$$
\left\{\begin{array}{l}
s_{2345}^{1}\left(c_{01}^{1} \pm c_{01}^{6}\right)=s_{2345}^{1}\left(\cos \left(\alpha-90^{0}+\theta_{1}^{1}\right) \pm \cos \left(270^{0}-\alpha+\theta_{1}^{6}\right)\right) \\
s_{2345}^{1}\left(s_{01}^{1} \pm s_{01}^{6}\right)=s_{2345}^{1}\left(\sin \left(\alpha-90^{0}+\theta_{1}^{1}\right) \pm \sin \left(270^{0}-\alpha+\theta_{1}^{6}\right)\right) \\
s_{2345}^{2}\left(c_{01}^{2} \pm c_{01}^{5}\right)=s_{2345}^{2}\left(\cos \left(\beta-30^{0}+\theta_{1}^{2}\right) \pm \cos \left(210^{0}-\beta+\theta_{1}^{5}\right)\right) \\
s_{2345}^{2}\left(s_{01}^{2} \pm s_{01}^{5}\right)=s_{2345}^{2}\left(\sin \left(\beta-30^{0}+\theta_{1}^{2}\right) \pm \sin \left(210^{0}-\beta+\theta_{1}^{5}\right)\right) \\
s_{2345}^{3}\left(c_{01}^{3} \pm c_{01}^{4}\right)=s_{2345}^{3}\left(\cos \left(\gamma+30^{0}+\theta_{1}^{3}\right) \pm \cos \left(150^{0}-\gamma+\theta_{1}^{4}\right)\right) \\
s_{2345}^{3}\left(s_{01}^{3} \pm s_{01}^{4}\right)=s_{2345}^{3}\left(\sin \left(\gamma+30^{0}+\theta_{1}^{3}\right) \pm \sin \left(150^{0}-\gamma+\theta_{1}^{4}\right)\right)
\end{array}\right.
$$

According to the rotation range of revolute joint, the condition $s_{2345}^{i} \neq 0$ is satisfied only when the projection of branch chains on plane satisfy the relations when $\alpha=180^{\circ} 、 \beta=120^{\circ} 、 \gamma=60^{\circ}$ under the initial state. As shown in figure 4

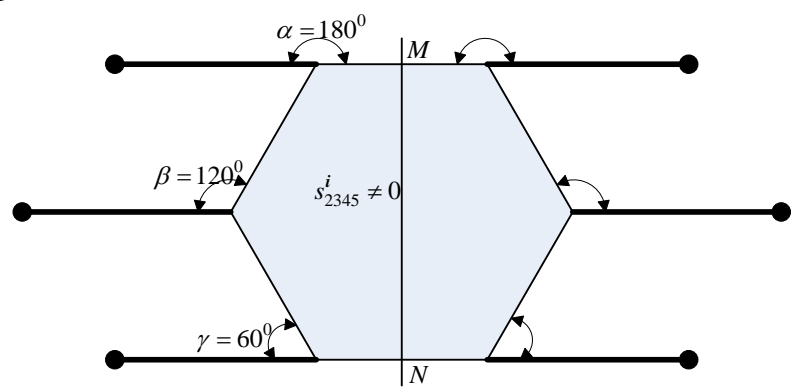

Figure 4:Initial state whit three DOF

The robot is in an state of 'unconventional' singular point under initial state when $s_{2345}^{i} \neq 0$. In the process of motion, if relation $s_{2345}^{i} \neq 0$ is satisfied, then the contraint $\theta_{1}^{i}=0$ must be also satisfied and $s_{2345}^{1}=s_{2345}^{2}=s_{2345}^{3}=-s_{2345}^{4}=-s_{2345}^{5}=-s_{2345}^{6}$. The matrix of spinors which express the constraint motion of body platform $A_{1} A_{2} A_{3} A_{4} A_{5} A_{6}$ is given as below:

$$
\begin{aligned}
& \xi_{A_{1} A_{2} A_{3} A_{4} A_{5} A_{6}}^{r}=\left[\begin{array}{cccccc}
\xi_{B_{1} A_{1}}^{\tau} & \xi_{B_{2} A_{2}}^{\tau} & \xi_{B_{3} A_{3}}^{\tau} & \xi_{B_{4} A_{4}}^{\tau} & \xi_{B_{5} A_{5}}^{\tau} & \xi_{B_{6} A_{6}}^{\tau}
\end{array}\right] \\
& =\left[\begin{array}{cccccc}
-s_{2345}^{1} & -s_{2345}^{1} & -s_{2345}^{1} & -s_{2345}^{1} & -s_{2345}^{1} & -s_{2345}^{1} \\
0 & 0 & 0 & 0 & 0 & 0 \\
0 & 0 & 0 & 0 & 0 & 0 \\
0 & 0 & 0 & 0 & 0 & 0 \\
c_{2345}^{1} M_{1} & c_{2345}^{1} M_{2} & c_{2345}^{1} M_{3} & -c_{2345}^{1} M_{4} & -c_{2345}^{1} M_{5} & -c_{2345}^{1} M_{6} \\
s_{2345}^{1} M_{1} & s_{2345}^{1} M_{2} & s_{2345}^{1} M_{3} & -s_{2345}^{1} M_{4} & -s_{2345}^{1} M_{5} & -s_{2345}^{1} M_{6}
\end{array}\right]\left[\begin{array}{l}
k_{1} \\
k_{2} \\
k_{3} \\
k_{4} \\
k_{5} \\
k_{6}
\end{array}\right]
\end{aligned}
$$

Normally $\operatorname{rank}\left(\xi_{A_{1} A_{2} A_{3} A_{4} A_{3} A_{6}}\right)=3$ and one group of motion spinors which present the spatial motion of the body platform can be determined as follows: $\xi_{\mathrm{A}_{1} \mathrm{~A}_{2} \mathrm{~A}_{3} \mathrm{~A}_{4} \mathrm{~A}_{3} \mathrm{~A}_{6}}=\left[\begin{array}{lll}k_{x} \xi_{1} & k_{y} \xi_{2} & k_{z} \xi_{3}\end{array}\right]$, where

$$
\left\{\begin{array}{l}
\xi_{1}=\left[\begin{array}{llllll}
1 & 0 & 0 & 0 & 0 & 0
\end{array}\right]^{T} \\
\xi_{2}=\left[\begin{array}{llllll}
0 & 0 & 0 & 0 & 1 & 0
\end{array}\right]^{T} \\
\xi_{3}=\left[\begin{array}{llllll}
0 & 0 & 0 & 0 & 0 & 1
\end{array}\right]^{T}
\end{array}\right.
$$


The DOF of end joints of platform is $M=\operatorname{rank}\left(\xi_{A_{1} A_{2} A_{3} A_{4} A_{3} A_{6}}\right)=3$. The robot has motion of translating along $y$ axis and $z$ axis and rotating around $x$ axis.

(2) The body platform has four DOF when $s_{2345}^{i}=0$.

Demonstration: If $s_{2345}^{i}=0$, then $c_{2345}^{i}=1$ and the matrix of spinors which express the constraint of motion of body platform $A_{1} A_{2} A_{3} A_{4} A_{5} A_{6}$ is given as below:

$$
\begin{aligned}
& \xi_{A_{1} A_{2} A_{3} A_{4} A_{3} A_{6}}^{r}=\left[\begin{array}{llllll}
\xi_{B_{1} A_{1}}^{\tau} & \xi_{B_{2} A_{2}}^{\tau} & \xi_{B_{3} A_{3}}^{\tau} & \xi_{B_{4} A_{4}}^{\tau} & \xi_{B_{5} A_{5}}^{\tau} & \xi_{B_{6} A_{6}}^{\tau}
\end{array}\right] \\
& =\left[\begin{array}{cccccc}
0 & 0 & 0 & 0 & 0 & 0 \\
0 & 0 & 0 & 0 & 0 & 0 \\
0 & 0 & 0 & 0 & 0 & 0 \\
c_{01}^{1} M_{1} & c_{01}^{2} M_{2} & c_{01}^{3} M_{3} & -c_{01}^{4} M & -c_{01}^{5} M_{5} & -c_{01}^{6} M_{6} \\
s_{01}^{1} M_{1} & s_{01}^{2} M_{2} & s_{01}^{3} M_{3} & -s_{01}^{4} M_{4} & -s_{01}^{5} M_{5} & -s_{01}^{6} M_{6} \\
0 & 0 & 0 & 0 & 0 & 0
\end{array}\right]\left[\begin{array}{c}
k_{1} \\
k_{2} \\
k_{3} \\
k_{4} \\
k_{5} \\
k_{6}
\end{array}\right]
\end{aligned}
$$

Normally $\operatorname{rank}\left(\xi_{\mathrm{A}_{1} \mathrm{~A}_{2} \mathrm{~A}_{3} \mathrm{~A}_{4} \mathrm{~A}_{3} \mathrm{~A}_{6}}\right)=2$ and one group of motion spinors which present the spatial motion of the body platform can be determined as follows: $\xi_{\mathrm{A}_{1} \mathrm{~A}_{2} \mathrm{~A}_{3} \mathrm{~A}_{4} \mathrm{~A}_{3} \mathrm{~A}_{6}}=\left[\begin{array}{llll}k_{1} \xi_{1} & k_{2} \xi_{2} & k_{3} \xi_{3} & k_{4} \xi_{4}\end{array}\right]$, where

$$
\left\{\begin{array}{l}
\xi_{1}=\left[\begin{array}{llllll}
0 & 0 & 0 & 1 & 0 & 0
\end{array}\right]^{T} \\
\xi_{2}=\left[\begin{array}{llllll}
0 & 0 & 0 & 0 & 1 & 0
\end{array}\right]^{T} \\
\xi_{3}=\left[\begin{array}{llllll}
0 & 0 & 0 & 0 & 0 & 1
\end{array}\right]^{T} \\
\xi_{4}=\left[\begin{array}{llllll}
0 & 0 & 1 & 0 & 0 & 0
\end{array}\right]^{T}
\end{array}\right.
$$

The DOF of end joints of platform is $M=\operatorname{rank}\left(\xi_{\mathrm{A}_{1} \mathrm{~A}_{2} \mathrm{~A}_{3} \mathrm{~A}_{4} \mathrm{~A}_{3} \mathrm{~A}_{6}}\right)=4$. The robot has motion of translating along three axes and rotating around $z$ axis. Obviously, the conditions when $\alpha \neq 180^{\circ}$ or $\beta \neq 120^{\circ}$ or $\gamma \neq 60^{\circ}$ under the initial state satisfy the requirement. According to conclusion(1) the robot is also on a singular state when $s_{2345}^{i}=0$ and $\alpha=180^{\circ} 、 \beta=120^{\circ} 、 \gamma=60^{\circ}$.

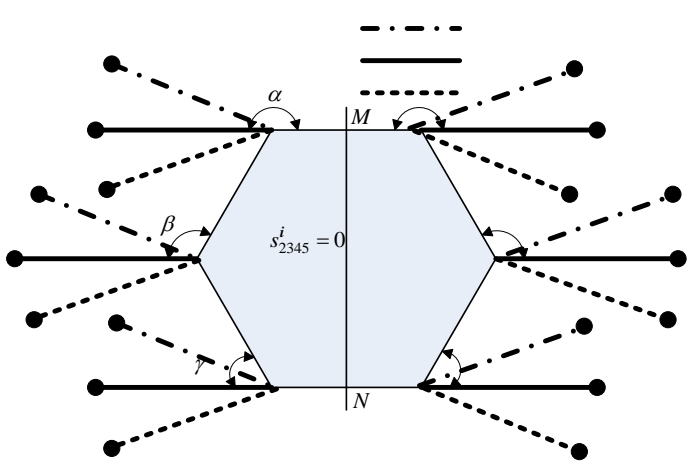

Figure 5:Initial state four DOF

(3) The robot is in "unconventional" singular state when $\alpha=180^{\circ} 、 \beta=120^{\circ} 、 \gamma=60^{\circ}$.

Demonstration: When the initial state satisfy $\alpha=180^{\circ} 、 \beta=120^{\circ} 、 \gamma=60^{\circ}$, based on the analysis above, the rank of matrix of spinors which express the constraint of motion of body platform under initial state is $N=\operatorname{rank}\left(\xi_{\mathrm{A}_{2} \mathrm{~A}_{2} \mathrm{~A}_{3} \mathrm{~A}_{4} \mathrm{~A}_{5}}\right)=1$. The robot has five DOF in this situation and has motion of translating along three axes and rotating around $\mathrm{x}$ axis and $\mathrm{z}$ axis. However, when robot leave this initial state, the DOF of robot will change into conclusion (1) or conclusion (2) which has three or four DOF. In this paper we refer it as "unconventional" singular state.

\section{Analysis of DOF under Initial States of Rotational Symmetry}

When initial state is rotationally symmetrical about line $O O_{c}$, the length of a side of body platform is defined as $R_{1}$. The projected of $B_{i} A_{i}$ on plane oxy is $R_{2}$. The coordinate values of foot tips are:

$$
B_{1}\left(-\frac{\sqrt{3}}{2} R_{1}-R_{2} \sin \vartheta,-\frac{1}{2} R_{1}+R_{2} \cos \vartheta, 0\right), \quad B_{2}\left(-R_{2} \sin \left(\vartheta-120^{\circ}\right),-R_{1}-R_{2} \cos (\vartheta-120), 0\right),
$$




$$
\begin{gathered}
B_{3}\left(\frac{\sqrt{3}}{2} R_{1}+R_{2} \sin \left(\vartheta-60^{\circ}\right),-\frac{1}{2} R_{1}-R_{2} \cos (\vartheta-60), 0\right) B_{4}\left(\frac{\sqrt{3}}{2} R_{1}+R_{2} \sin (\vartheta), \frac{1}{2} R_{1}-R_{2} \cos (\vartheta), 0\right), \\
B_{5}\left(-R_{2} \sin \left(120^{0}-\vartheta\right), R_{1}+R_{2} \cos \left(120^{0}-\vartheta\right), 0\right) B_{6}\left(-\frac{\sqrt{3}}{2} R_{1}-R_{2} \sin \left(240^{0}-\vartheta\right), \frac{1}{2} R_{1}-R_{2} \cos \left(240^{\circ}-\vartheta\right), 0\right)
\end{gathered}
$$

The constraint relations of body platform $A_{1} A_{2} A_{3} A_{4} A_{5} A_{6}$ is similar to section 3.1 and the matrix of constraints of body platform $A_{1} A_{2} A_{3} A_{4} A_{5} A_{6}$ is given below:

$$
\xi_{A_{1} A_{2} A_{3} A_{4} A_{3} A_{6}}^{r}=\left[\begin{array}{llllll}
\xi_{B_{1} A_{1}}^{\tau} & \xi_{B_{2} A_{2}}^{\tau} & \xi_{B_{3} A_{3}}^{\tau} & \xi_{B_{4} A_{4}}^{\tau} & \xi_{B_{5} A_{5}}^{\tau} & \xi_{B_{6} A_{6}}^{\tau}
\end{array}\right]
$$

Where

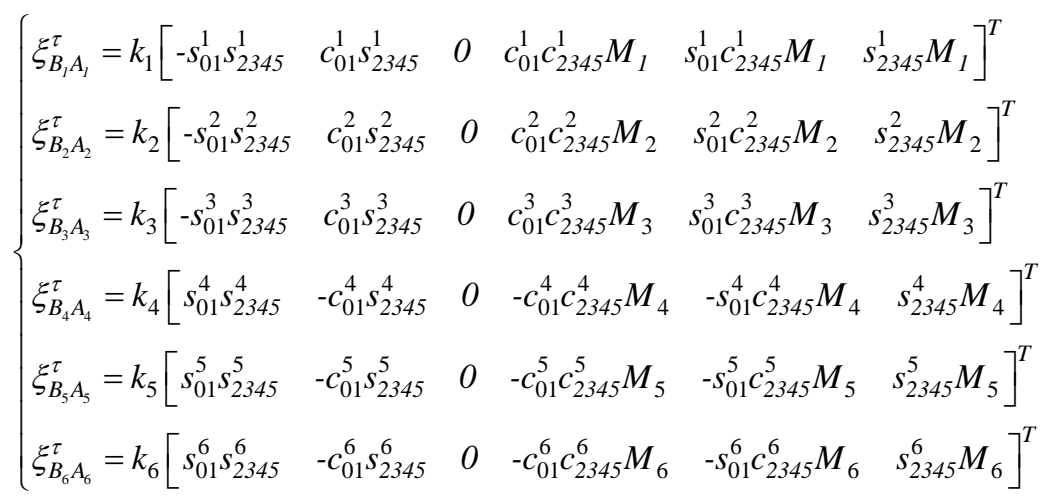

The $k_{i}$ is an arbitrary non-zero constant and $\theta_{01}^{1}=\alpha-90^{0}+\theta_{1}^{1}, \theta_{01}^{2}=\alpha-30^{0}+\theta_{1}^{2}, \theta_{01}^{3}=\alpha+30^{0}+\theta_{1}^{3}, \theta_{0}^{4}=\alpha-90^{0}+\theta_{1}^{4}, \theta_{01}^{5}=\alpha-30^{0}+\theta_{1}^{5}, \theta_{01}^{6}=\alpha+30^{0}+\theta_{1}^{2}$. Clearly, $\xi_{A_{1} A_{2} A_{3} A_{1} A_{5} A_{6}}$ is related with initial states and the rotation angle of each joint. The conclusion is given as follows.

(1)The DOF of body platform in different initial states is four.

Demonstration: When $s_{2345}^{i} \neq 0$, the corresponding constraints in section 3.1 have to be satisfied. Therefore according to the analysis of last section, the motion of the body platform has no solution when $s_{2345}^{i} \neq 0$, which means that $s_{2345}^{i}=0$ in this condition. Besides, according to the second conclusion in section3.1, the DOF of the robot is four. The robot has motion of translating along three axes and rotating around $z$ axis. The initial state of robot is shown in figure 6 .

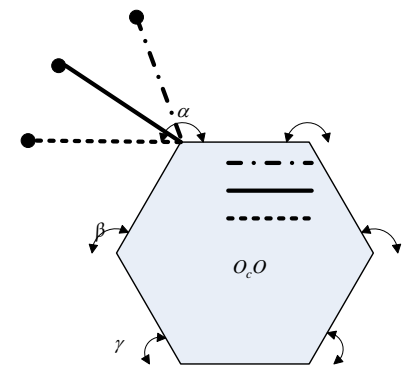

Figure 6:Initial states of rotational symmetry

From the analysis above, the DOF of robot is changes under different initial states. According to the requirement, initial states must be designed firstly. It also shows that workspace of robot is related to initial states. 


\section{Summary}

Based on the spinor theory, the DOF information, especially the DOF information under symmetrically initial states are discussed in detail. The method to calculate DOF from motion matrix of platform is more applicable and simpler than the traditional G-K method.

Hexapod wall-climbing robots have "unconventional" singular state because of its special structure. In that state the DOF of robot is not only determined by structure and initial state, but also determined by the subsequent motion when the robot leave the initial state. This special feature of hexapod robots must be concerned in trajectory planning and design of gait and is worth to be deeply analyzed.

\section{Acknowledgements}

Corresponding Author: Wei Wu, eeweiwu@126.com

Supported by the National Natural Science Foundation of China (Grant No. 61573148); Major projects of Guangdong (Grant No. 2015B010919007);

\section{References}

[1] L.W. Tsai, G.C. Walsh, R.E. Stamper, Kinematics of a novel three DOF translational platform. Proc of 1996 IEEE Int. Conf. on Robotics and Automation. Minneapolis, Minnesota, April, 1996:3446-3451

[2] H.S. Kim, L.W. Tsai, Design Optimization of a Cartesian Parallel Manipulator. ASME DETC2002/MECH-34301, 2002

[3] Eckhardt H D. Kinematic Design of Machines and Mechanisms. New York: McGraw-Hill, 1999: 20-37

[4] G. Gogu, Mobility of mechanisms: A critical review. Mech. Mach. Theory, 2005, 40: 1068-1097

[5] J.M. Rico, J. Gallardo, B. Ravani, Lie algebra and the mobility of kinematic chains. J. Rob. Syst, 2003, 20: 447-499

[6] R. Alizade, C. Bayram, Structural synthesis of parallel manipulators. Mech. Mach. Theory, 2004, 39: $857-870$

[7] J.S. Zhao, K. Zhou, Z.J. Feng, A theory of degrees of freedom for mechanisms. Mech. Mach. Theory, 2004, 39(6): 621-643

[8] Z. Huang, Y.S. Zhao, T.S. Zhao, Advanced Spatial Mechanism[M], Beijing: Higher Education Press, 2006

[9] X.W. Kong, C.M. Gosselin, Mobility Analysis of Parallel Mechanisms Based on Screw Theory and the Concept of Equivalent Serial Kinematic Chain. ASME DETC-85337, 2005

[10] C.B. Wampler, T.A. Larson, A new mobility formula for spatial mechanisms and its Application. ASME DETC2007-335574, 2007

[11] J.M. Rico, et al, Mobility and over constraint in kinematic chain and assemblies. ASME DETC2007-34937, 2007

[12] J.S. Zhao, Z.J. Feng, K. Zhou, et al, Re-analysis on the degree of freedom of the platforms in spatial parallel mechanisms with constraints spaces. The International Journal of Advanced manufacturing Technology, 2006, (1,2): 190-196

[13] J.S. Zhang, Z.J. Feng, L.P. Wang, et al. The free mobility of a parallel manipulator. Robotica, 2006, (5): 635-641 